\title{
AN EXPERT SYSTEM TO OPTIMIZE COST AND SCHEDULE OF HEAVY EARTHMOVING OPERATIONS FOR EARTH- AND ROCK- FILLED DAM PROJECTS
}

\author{
Nizar MARKIZ, Ahmad JRADE \\ Department of Civil Engineering, University of Ottawa, Ottawa, ON, Canada, K1N 6N5
}

Received 03 Oct 2014; accepted 23 Feb 2015

\begin{abstract}
Success of major embankment dam construction projects is measured by the enormity of optimizing costs and schedules of selected heavy equipment based on their operational analyses. In this paper, the main objective is geared towards developing a knowledge-based decision support system for optimizing costs of heavy earthmoving operations and corresponding linear schedules at early design stages. Also, the proposed system is capable of generating an automated linear schedule based on stochastic scheduling techniques. Thus, a meta-heuristic simulated approach utilizing a metropolis algorithm is implemented to assist in generating optimized line-of balances. The successful implementation of the proposed system will provide the user with optimum fleet of equipment for performing earthwork operations and linear scheduling for strategic planning purposes. Towards the end, an actual dam construction project is utilized to numerically validate the proposed system and quantify its degree of accuracy. Results presented in this study are anticipated to be of major significance to owners, designers, and construction managers specialized in embankment dams heavy earthmoving operations and would contribute to the database of fleet management systems by incorporating a novel system that integrates heavy equipment economical operational analyses with its corresponding line of balance.
\end{abstract}

Keywords: decision-support, heavy equipment, operation analysis, cost estimation, linear scheduling.

\section{Introduction}

Construction of embankment dam projects significantly differs from one to the other. There is no such perfect method that can estimate optimum fleet. However, technological advancements that have been made so far in planning and analyzing construction operations are increasingly assisting owners, designers, and construction managers in evaluating different alternatives and selecting optimum fleet. In general, earthmoving operations are undertaken based on a particular linear activity sequencing plan. Although such operations are unique in nature, their corresponding operational analyses are cyclic-repetitive. For instance, an earthmoving operation pertaining to the construction of a bridge foundation comprises excavation preceded by clearing and grubbing, loading, and hauling and concluded by backfilling. This process implies that the predecessor operation must be completed prior to the start of the successor while the overall work process is repetitive. Therefore, the cyclic-repetitive processes of heavy earthmoving operations are capable of being modeled as discrete event simulations. In this study, discrete event simulations based on heavy earthmoving equipment comprehensive operational analyses has proven its reliability in modeling cyclic-repetitive processes based on logical relations among operations, scope-of-work related parameters, and availability of resources.

The expert system presented herein comprises a knowledge base system and a hypothetical reasoning inference engine based on a forward chaining engine technique. The expert system represents factual information pertaining to heavy earthmoving operations of dams (i.e. static and dynamic parameters; besides working variables) where an inference engine is employed to evaluate the knowledge-base and deploy subsequent numerical models and constraints to explicitly infer new results and further incorporate into the initial knowledge base. As part of the expert system, a hypothetical reasoning approach supported by a forward chain reasoning strategy to simultaneously explore solutions and provide explanations to the conclusions achieved.

\section{Objectives and methodology}

The main objective of this research incentive is to develop a decision support system utilizing a linear cost and schedule optimization approaches for heavy equipment selection in order to assist owners, designers, and construction managers in this multifaceted task. The system design presented in this paper provides the user with 
an optimum fleet that balances interdependent equipment such as the loading-hauling system. To achieve this objective, the following steps are considered: (1) identifying factors that influence equipment selection; (2) classifying earthwork activities; (3) selecting number of equipment for each type of earthwork activity; (4) analyzing the operations for each type of earthwork activity; (5) developing a system that predicts optimum number and size of equipment fleet based on their associated ownership and operating costs; (6) providing an automated optimized linear schedule based on calculated productivity rates; and (7) validating the proposed system with an actual project. The proposed research methodology comprises three areas as follows: (1) literature review; (2) system development; and (3) validation. The framework of the proposed methodology is to study the factors that influence equipment selection. At this stage, the idea is to obtain a wide understanding of how equipment selection is affected by these factors. Since the influence of these factors is numerous and complicated, it is important to study them comprehensively and to identify their impact on equipment selection. The proposed system components are illustrated in Figure 1.

\section{Literature review}

Numerous methods and models have been proposed to optimize equipment selection for different types of activities; however, the majority of the studies in the literature focus on the optimization of equipment selection in heavy civil work based on diverse complex factors; however, none of the studies are to include or perform economical operation analysis. One study conducted by Moselhi and Marzouk (2000) addresses cost estimation of heavy earthmoving operations. In their study, an equipment cost application system for time and cost estimation of heavy earthmoving operations is developed. The system is then verified by a numerical example with a detailed step-bystep description of the procedure to be followed. This study is of major significance at the design stage of a construction project and is limited to initial costs anticipated for earthmoving operations.

Another study conducted by Marzouk and Moselhi (2003) addresses cost applications without considering complex factors in heavy equipment operation analysis. In their study, an object-oriented simulation model for earthmoving operations is developed. The model is implemented in a Microsoft environment to enhance its components integration capabilities with the Visual Basic 6.0 code. The proposed model consists of a simulation program, a database and cost applications, and an optimization and reporting module. At the end, the study is verified with a numerical example by comparing the corresponding outputs of the Caterpillar's software to the developed earthmoving simulation program. It is concluded that results are in good agreement with a percentage difference less than $8 \%$. Also, it is found that simulation program is considered more accountable than the Caterpillar's software for uncertainties that arise during the execution of earthmoving operations. The main focus of their paper, however, is targeted towards the simulation program only. Moreover, a model based on an analytical hierarchy process is developed by Shapira and Goldenberg (2005). The model is intended to provide solutions for two main issues as follows: (1) systemic evaluation of soft factors; and (2) weighing of soft benefits when compared to costs.

Other studies have addressed significant factors that influence operation analysis. For instance, a fuzzy clustering model for estimating haulers' travel time capable of being integrated with diverse simulation and estimation models is developed by Marzouk and Moselhi (2004).

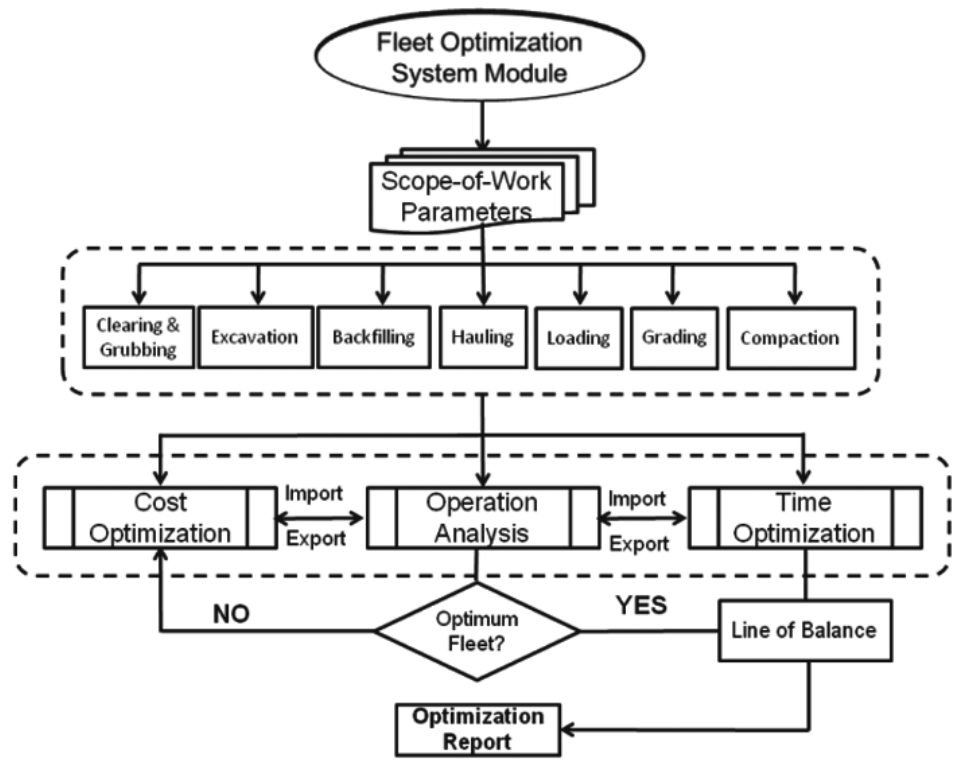

Fig. 1. System components 
The model exploits regression analysis and subtractive clustering Furthermore, a simulation model for optimization equipment selection in earth-fill dam projects based on a nonlinear optimization technique is developed by Rafsanjani (2011). The model proposed is intended to assist users in selection of optimum fleet of equipment with minimal time and cost parameters.

In a recent study conducted by Fu (2013), a simulation system for earthmoving operations to enhance overall productivity and performance is presented. The model is designed to confirm the optimality of a selected fleet by deploying discrete event simulation strategies to correlate interdependencies between equipment resources and earthmoving activities. These models are proposed for specific types of construction work due to the many factors that contribute to equipment selection; however, researchers have focused on developing expert systems to assist construction managers and builders in selecting optimum fleet, and, unfortunately, had not considered solutions comprising economical operation analysis integrated with equipment comprehensive owning and operating costs.

\subsection{Ownership costs}

Ownership costs are fixed and incurred costs whether the equipment is used or not. Ownership costs include: (1) purchase expense; (2) interest; (3) salvage value; (4) property taxes; (5) insurance; (6) license fees; and (7) depreciation. In this study, diverse depreciation numerical methods are deployed to estimate the loss in value at the end of any specific period.

\subsection{Operating costs}

Operating costs are costs associated with operating the equipment and variable in terms of the total cost. These costs include: (1) fuel; (2) lubricants; (3) filters; (4) hydraulic fluids; (5) parts; (6) maintenance; (7) repairs reserve; (8) tires cost and repairs; (9) special wear items; and (10) and operator wages.

\subsection{Data collection}

The most important step in analyzing equipment operations resides in understanding the characteristics of the materials to be moved, which in turn affects the type of equipment required to successfully complete the project. Therefore, diverse material properties are extracted from Caterpillar ${ }^{\circledR}$ Performance Handbook (2011) and are inherited into the developed system. Also, mathematical formulations pertained to the operation analysis are incorporated into a set of modules to conclude the data collection phase. Some of these data are tabulated in order to enhance the system capability of interfacing the data with the information that has to be entered by the user. Furthermore, the database of information incorporated into the modules possess a variety in the equipment capacity, power, and maximum allowable weight which enables the developed system to be applied for any construction project regardless of the volume and types of materials involved.

\section{Linear scheduling}

Linear scheduling is a technique utilized in heavy civil work projects and known to be a unique mean of resource allocation for earthmoving operations with linear curves display of time-space interface. Usually, implementation of linear scheduling is well appropriate for horizontal construction projects that comprise concurrent and repetitive operations. Linear scheduling represents a particular earthmoving activity as a linear production curve such that station/location and time are on the $y$ - and $\mathrm{x}$-axes respectively; and are interrelated in a 2D graphical display. Typically, production lines are shown as linear curves with constant slopes; however, in practical situations, a particular activity features inconsistent slopes due to dynamic production rates attributed to specified large quantity, level of work complexity, erratic weather conditions and job-related circumstances.

\subsection{Stochastic linear scheduling process}

The stochastic linear scheduling methodology proposed in this study is capable of modeling fluctuations in earthmoving activity performance based on productivity rates calculated from actual project data. In order to achieve this goal, actual project data is extracted from the fleet productivity optimization module and further analyzed by inputting the data into a simulation system to assist the user in quantifying variations in an earthmoving activity productivity rate. The simulated system then analyzes generated productivity rates by fitting them to a standard Gaussian distribution and determining the bestfit. This allows the scheduler to view a probabilistic representation of overall earthmoving activities performances. Furthermore, in one study conducted by Rachmat et al. (2012), it is concluded that standard distributions are proven to outperform other distributions for linear construction operations. Moreover, in their study, the authors setup experiments utilizing Chi-square and the Kolmogorov-Smirnov tests to prove the same. Therefore, in this study and based on the afore-mentioned, standard Gaussian distributions are deployed.

\subsection{Probabilistic distribution fitting}

The initial step when modeling probabilistic distribution fittings is to develop a generic histogram pertaining to activity productivity rates. Based on the characteristic shape of the generated histogram; an indication of a standard probabilistic distribution that will result in a "best-fit" fitting will be evident. Furthermore, corresponding distribution parameters, such as the mean and standard deviation may be determined from the probabilistic fitting. It is important to note that due to the technological advancement in probabilistic distribution modeling software, an 
indefinite number of distribution may be automatically fitted within a reasonable time frame. However, a selective number of candidate distributions are typically forwarded to quality of fit testing procedure prior to engagement in further processes.

\subsection{Quality of fitting}

Although multiple standard distributions are capable of modeling a probabilistic fitting, significant variance may be noticed in the manner they represent the actual distribution underlying histogram data set. Testing the quality of fit for a distribution is conducted by either of the two following procedures: 1) heuristic, where manual inspection is conducted in parallel with an error minimization procedure; or 2) non-heuristic procedures, where hypothetical procedures such as the Chi-square test are deployed. In order to ease the use of distribution modeling amongst owners and designers, the manual inspection of distribution fitting with an error minimization procedure is adopted since distribution fittings are automatically generated with advanced modeling software available in the market. In this study, productivity rate histograms are normalized for comparison purposes with the suggested scaled distribution. As part of enhancing the quality of a fit, an error minimization procedure is proposed for the curve-fitting of the suggested distribution to the actually obtained one. The procedure is based on applying a scaling factor to the suggested. The sum $E$ of the squares of differences between the actual and suggested fit is then minimized to obtain the magnitude of factor that results into the best fit with actual project data. The error minimization scheme proposed is summarized in Eqn (1):

$$
E=\sum_{i=1}^{n}\left[\frac{p_{a c t, i}-a\left(p_{s u g_{i}}\right)}{\bar{p}_{a c t, i}}\right]^{2}=\min ,
$$

where: $i=1, \ldots, n$ are the number of actual productivity rates, $p_{\text {act }, i}$ is the actual productivity rate at the $i^{\text {th }}$ location, $p_{\text {sug }}$ is the suggested productivity rate at the $i^{\text {th }}$ location, $a$ is a scaling factor to be applied to the suggested productivity rate values. It is noted that the bracketed terms in Eqn (1) have been normalized with respect to the average actual productivity rate, $\bar{p}_{a c t, i}$ as per Eqn (2):

$$
\bar{p}_{a c t, i}=\frac{1}{n} \sum_{i=1}^{n} p_{a c t, i} .
$$

Towards the end, it is important to note that the suggested productivity rates distribution fit provide a good representation of a logically sequenced line of balance where time and space buffers are respected.

\subsection{Estimating activity duration}

Estimating the duration of earthmoving activity is a multifaceted task that faces owners, designers, and construction managers as the occurrence of unforeseen events is very probable and unpredictable. In this study, a sto- chastic linear scheduling technique that encompasses the capability of modeling the variability among the activity performance-related factors that significantly affect productivity data is proposed as follows: (1) weather; (2) learning curve; (3) overtime; (4) space congestion; and (5) design changes. The value of these parameters is incorporated into the decision matrix and their calculations are automatically performed by the system. In order to provide a simulation input for a scheduler, an optimization system developed earlier by the authors of this study for the selection of optimum fleet based on their productivity rates is utilized to implement into the developed linear scheduling technique (Jrade et al. 2012; Jrade, Markiz 2012). Earthmoving activities productivity rates and corresponding durations are automatically extracted from the fleet productivity optimization module database in order to generate a histogram where a statistical normal distribution is utilized to "best-fit" the data set by utilizing the Gaussian Eqn (3):

$$
f(x, \mu, \sigma)=\frac{e^{-\frac{(x-\mu)^{2}}{2 \sigma^{2}}}}{\sigma \sqrt{2 \pi}},
$$

where: $x$ is a random variable, $\mu$ is the mean, and $\sigma$ is the standard deviation. The process begins by normalizing the normal distribution and histogram curves for "goodness-of-fit" comparison purposes. Afterwards, distribution parameters are determined via a trial and error process throughout the fitting process. Then, the expected duration of each activity is calculated by using the following Eqn (4):

$$
E=\frac{(O+4 M+P)}{6}
$$

where: $E$ is the expected duration time, $O$ is the optimistic duration time, $M$ is the most likely duration time, and $P$ is the pessimistic duration time. Once the expected duration time for each of the earthmoving activities is determined, a linear schedule optimization approach is employed.

\section{System conceptual modeling}

The development of the system comprises the following four main phases: (1) data collection; (2) operational analysis; (3) cost estimation; and (4) linear scheduling. As part of collecting data, a database of equipment specifications and scope of work related parameters is created. Then, a set of equipment operational analysis formulations as specified in the Caterpillar ${ }^{\circledR}$ Performance Handbook (2011) are utilized to assist in estimating equipment productivity based on project duration and specified scope of work while satisfying all equipment-related constraints. Following the equipment operational analysis, an economic analysis based on comprehensive owning and operating costs is conducted for all equipment based on their corresponding production rates obtained from the previous phase. After that, an equipment fleet is selected based 
on a linear cost optimization approach. A final optimum report, providing equipment ownership and/or rental options, is then presented to the user. A detailed description of the four main phases of the proposed system is described in the next paragraphs.

\subsection{Linear scheduling optimization}

In order to properly optimize logistics linear scheduling, one must understand all related constraints; otherwise, failure to do so may lead to erroneous results. In scheduling terms, deployment of optimum available resources is a multifaceted task for constructions managers. In this study, a linear scheduling optimization module is proposed to optimize the use of available resources by optimizing heavy fleet productivity by selecting most suitable equipment for an earthmoving activity. The module utilizes simulated annealing as an optimization tool supported by a classical constrained-based simulated algorithm, which indicates that linear scheduling constraints must be satisfied prior to the commenced of the associated task. The development of simulated annealing approach is described in details in the latter.

\subsubsection{Constraint-based simulation}

Modeling linear scheduling simulations as constraint satisfaction problems is a thoughtful pattern capable of solving diverse scheduling conflicts. According to Rossi et al. (2006), constraint-based simulation problems are typically defined by a set of variables and corresponding constraints. It is important to note that constraints parameters, specified among a defined set of variables, must be satisfied for one activity prior to proceeding to the next one. Beißert et al. (2007) summarized few constraints for typical construction projects as follows: 1) technological advancements; 2) equipment capacity; 3) availability of resources; and 4) logistical aspects. Technological advancements pertaining to earthmoving equipment may be categorized as follows: 1) equipment implements; 2) traction systems; 3) structure and suspension systems; 4) power train systems; and 5) control and information systems. While logistical aspects constraints of equipment are summarized as follows: 1) time and cost for mobilizing and demobilizing crews; 2) use of equipment in preceding operations and parked time; and 3) support facilities. In addition to that, for heavy earthmoving operations, the two major constraints that must be satisfied are: 1) time- and 2) space- related constraints, which are typically referred to as time and space buffers respectively. In this study, research efforts to enhance the discrete event simulation technique have been utilized such that constraint satisfaction problems are integrated with a simulated annealing approach such that interdependencies among tasks; i.e. hauling-loading are taken into consideration and results are obtained in a timely manner. In simulated annealing processes, the presumed constraint is always satisfied and controlled within the procedure itself. In other words, no event or task can be scheduled without its time and space buffers being met which produces a logically sequenced linear schedule where further analysis to time and space constraints may be conducted.

\subsubsection{Simulated annealing system process}

In this context, a meta-heuristic metropolis algorithm is deployed as a local optimization approach to resolve linear scheduling combinational conflicts and reach an optimum solution within reasonable cycle time. According to Dreo et al. (2006), the annealing process begins when metal particles unveil their original state and re-configure into a highly organized structure with lower energy than the initial state. In our case, the simulated annealing procedure may be comprehended as the methodology employed to reach at an optimum solution which represents the possibility of new configurations of the metal particles. Simulated annealing is usually implemented by assuming a high initial temperature and meta-heuristically determines a new solution within the neighborhood of the initial solution. It is important to note that the probability of acceptance of re-configured solutions is dependent upon the difference between immediate consecutive solutions $(\varepsilon)$ and temperature $(\alpha)$. Once accepted, the new solution is set as the starting point for the consecutive optimization cycle. Consequently, in order to implement the generic metropolis algorithm, the following criterions must be met: (1) suitable neighborhood; (2) proper probability of acceptance; and (3) effective temperature decreasing rate in order to reach a successful optimum linear schedule and consequently escape local minima. Towards the end, simulated annealing is integrated into the constraint-based simulation in an attempt to enhance neighboring schedules by substituting tasks. Once an optimized schedule is determined, the newer solution replaces the older one and simultaneously enables formerly declined solutions to be accepted in order to escape local minima.

\subsubsection{Neighborhood sequencing}

In simulated annealing scheduling approaches, the distinctive concept of local "neighbor-hooding" is crucial. Conceptually, a schedule possesses a local neighborhood only when two activities of the same ranking are substituted. Typically, tasks ranking are determined through a complex topological sorting technique. The concept of topological sorting is based on transforming a partial order to a total order. For instance, if $a>b$ and $b>c$, then it implies that $a>c$. However, $a$ and $b$ may be neither $a>b$ nor $b>a$. A total order may be achieved when either $a>b$ or $b>a$ for all $a \neq b$. Hence, the system proceeds by generating multiple solutions by substituting tasks of local neighborhood with similar rankings. Once a solution is generated, neighborhood tasks are assigned ranks along with a corresponding execution order for each ranked task. For instance, activities A and B may be assigned rank one; however, task B may precede task A in terms of order of execution. In the next step, tasks 
C and D of rank two maybe be substituted to form an alternative solution and so forth.

\subsubsection{Probability of acceptance}

Rules of accepting a solution within a simulated annealing process is a crucial element of the generalized metropolis algorithm. In order to illustrate probability of acceptance process, a system in its current state described by an $\mathrm{N}$-dimensional vector $\mathrm{x}$ comprising a probability function $f(t)$ is defined. According to Gould and Tobochnik (2010), a set of values that control the convergence speed of an annealing algorithm must be defined. Typically, these values can never be predicted at the initial stage. Instead, the values depend on the type of optimization problem and must be adjusted accordingly. Therefore, the process commences upon the selection of initial configuration, $x$. Then, the initial number of simulations must be set to zero, $n_{S}=0$. Afterwards, an initial temperature must be set to some high value, $t_{o}$. This step is considered to be one of the most significant steps as the convergence of the simulated annealing algorithm is dependent on the selection of initial temperature, $t_{o}$, cooling parameter, $\alpha$ and decreasing rate, $\Delta t$. Within the scope of this study, the decreasing rate of the initial temperature is determined as per Eqn (5):

$$
\Delta t=t_{i}(1-\alpha),
$$

where: $\Delta t$ is the decreasing rate, $t_{i}$ is the current temperature, and $\alpha$ is the cooling parameter. Based on earlier studies, typical values of the cooling parameter, $\alpha$, vary between 0.8 and 0.999 with the latter being the closest to ideal. Prior to decreasing in temperature, an initial value of the probability function must be obtained as per Eqn (6):

$$
f_{a}=f\left(t_{i}\right) .
$$

Then, a second value is determined following the transition as per Eqn (7):

$$
f_{b}=f\left(t_{i}-\Delta t\right) .
$$

Afterwards, the difference between the obtained values of the probability function is determined according to Eqn (8):

$$
\Delta f=f_{b}-f_{a} .
$$

The following step involves an "if-then" loop console where if, $\Delta f \leq 0$, the current state is accepted; otherwise, a generic value, $\varepsilon$, is the value at which the algorithm is terminated. In this study, $\varepsilon$ is assigned a value of 0.001 based on trial and error. The rule of accepting the state of function is then defined as per Eqn (9):

$$
\varepsilon<e^{\frac{-\Delta f}{t_{i}}}
$$

where: $\varepsilon$ is the value at which the algorithm is terminated, $\Delta f$ is the change in probability functions values,

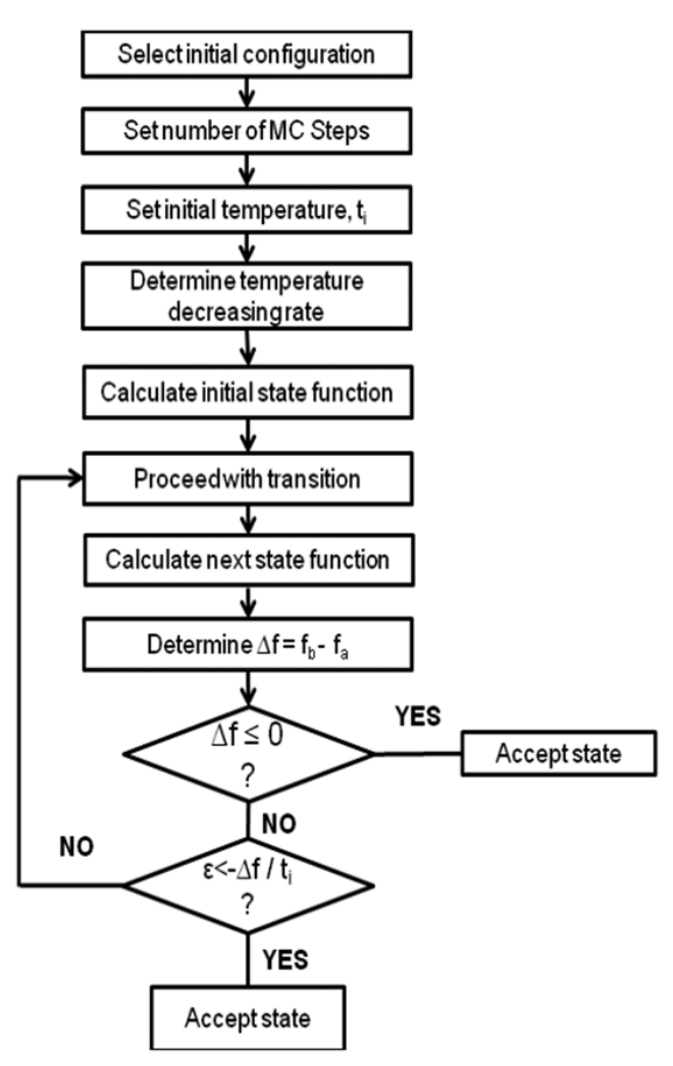

Fig. 2. Procedure for rule of accepting a function state

and $t_{i}$ is the current temperature. However, if the state of the function is rejected, then the process is directed to another transitional iteration, $n_{S}=n_{S}+1$ until the initial temperature reaches the value of zero. The detailed procedure for rule of accepting a function state is shown in Figure 2.

\section{Global optimization}

Global optimization is a numerical analysis procedure that optimizes an objective nonlinear function by determining the best solution globally. Global optimization is known for its capability in bypassing local optima and seeking a global solution of a boundary-constrained objective function. In this study, research efforts are focused to enhance linear scheduling optimization a step further by enhance optimizing linear schedule by proposing a global optimization procedure in order to combine the expected durations of neighborhood activities of similar productivity rates while respecting time and space buffer constraints. In order words, activities of similar slope on a line of balance may be combined into a single task by integrating their corresponding time interdependencies together.

\section{System physical implementation}

The system is developed in an object-oriented .NET framework "C\#" while utilizing, SQLite, which is found to resolve interoperability issues among the "sub-data- 
base" applications within the developed prototype. The main purpose of the system is to facilitate the interface between operational analyses, user input data, and optimization functions. The modules are organized by activity names where every module has four sub-modules. The modules are as follows: (1) an operation analysis module that contains equipment operation calculations; (2) an economic analysis module that includes all owning and operating costs-related parameters and calculations; (3) an optimization module that displays optimization results; and (4) a report module which contains professional output reports that summarize results extracted from the optimization module. The system development process is detailed in the succeeding paragraphs. At first, the developed system is designed to automatically extract information entered by the user (i.e. volume of earthmovings, piling, backfilling, and subsequent grading and compaction). Then, the user would select the required module that correspond to the earthwork activity (i.e., clearing and grubbing, excavation, loading, hauling, backfilling, grading, compacting) from a user-friendly gateway. At this time, the user must enter job specific data required to execute necessary calculations. It is important to note that the data required for the economical operation analysis vary from one earthwork activity module to the other; however, (1) material density; (2) fill factors; (3) safe factors; (4) time constraints; (5) amount of work; and (6) operational efficiency is a common list of data variables among all seven activity modules. The amount of work to be undertaken is measured in terms of volume of earth material in loose cubic yard and is taken into account as a user-input parameter which is in turn used by the economical operation analysis module to obtain the optimum fleet. At last, the user is guided to the next phase where cost factors are to be entered. If any of the constraints set by the system are not satisfied, an error message pops up to inform the user that some equipment will not be considered in the optimization process. Afterwards, the user is directed towards fleet operation analysis calculations. When completed, the user must click on owning and operating cost link to complete the optimization process where an optimization button is to be clicked on for optimum fleet results. Towards the end, the developed prototype recalls the obtained productivity rates from the fleet optimization module for the diverse activity types. The rates are then organized into a histogram as described earlier where a statistical normal distribution is fitted accordingly and followed by the stochastic linear scheduling process. Once completed, an optimized line-of-balance is established throughout a simulated annealing approach accompanied by a global optimization objective function where time and space buffers conflicts are automatically flagged. The line of balance is obtained by automatically extracting corresponding productivity rates of ranked activities and plotting them against the calculated expected duration as per Eqn (3). Afterwards, the activity rankings are represented graphically with duration being on the y-abscissa and activity being on the x-abscissa. An optimization approach is then utilized to graphically fit the dataset into a regression function to determine a global local minima and maintain a balanced operational process flow. Once completed, adjacently ranked activities duration period are combined to reach at an optimum solution. The user is then privileged to modify activity durations to resolve conflicts and reach at an optimum linear schedule.

\section{System validation}

To validate the workability of the developed system, an actual project, which consists of the construction of a dam for a total cost of $\$ 2$ billion, was recalled from the literature (Marzouk, Moselhi 2004). The challenge underlying the system validation is to determine the optimum fleet necessary to execute the construction of the dam. The project was phased into three stages, each at different elevation and spanning a complete construction season. Table 1 summarizes the scope of earthwork fill at each operational stage.

Table 1. Scope of earthwork fill (BCY) at three different operational stages (Marzouk 2002)

\begin{tabular}{llcll}
\hline Soil Type & $\begin{array}{c}\text { Stage 1 } \\
\text { Elevation } \\
(1027 \mathrm{ft})\end{array}$ & $\begin{array}{c}\text { Stage 2 } \\
\text { Elevation } \\
(1237 \mathrm{ft})\end{array}$ & $\begin{array}{c}\text { Stage 3 } \\
\text { Elevation } \\
(1345 \mathrm{ft})\end{array}$ & $\begin{array}{c}\text { Total } \\
(\mathrm{BCY})\end{array}$ \\
\hline Moraine & 38,192 & 727,029 & 353,017 & $1,118,238$ \\
Granular & 18,965 & 374,729 & 181,806 & 575,500 \\
Rock & 257,929 & $4,197,751$ & $2,096,521$ & $6,555,201$ \\
Total & $\mathbf{3 1 5 , 0 8 6}$ & $\mathbf{5 , 2 9 9 , 5 0 9}$ & $\mathbf{2 , 6 3 1 , 3 4 4}$ & $\mathbf{8 , 2 4 5 , 9 3 9}$ \\
\hline
\end{tabular}

Prior to inputting data, a list of assumptions is made based on normal job conditions as follows: (1) gear efficiency $=0.85 ;$ (2) operational efficiency $=50(\mathrm{~min} / \mathrm{hr})$; (3) job conditions = average; (4) altitude $=2,500 \mathrm{ft}$; and (5) tire penetration $=3 \mathrm{in}$. It is important to note that results are obtained with time constraints. The assumptions contribute towards the difference noted between actual project and system data. Table 2 summarizes results for the three different material types (moraine, granular, and rock) earthmoving operations activity phases at different stages as extracted from the fleet optimization system.

Table 2. Comparison of system fleet results with actual project data

\begin{tabular}{llccc}
\hline & Soil Type & Stage 1 & Stage 2 & Stage 3 \\
\hline System Data & Moraine & $(2,1)^{\mathrm{a}}$ & $(4,1)^{\mathrm{a}}$ & $(4,1)^{\mathrm{a}}$ \\
& Granular & $(3,1)^{\mathrm{a}}$ & $(3,1)^{\mathrm{a}}$ & $(3,1)^{\mathrm{a}}$ \\
& Rock & $(2,1)^{\mathrm{a}}$ & $(4,1)^{\mathrm{a}}$ & $(3,1)^{\mathrm{a}}$ \\
\hline Actual Project & Moraine & $3,1)^{\mathrm{a}}$ & $(12,2)^{\mathrm{a}}$ & $(5,1)^{\mathrm{a}}$ \\
Data & Granular & $3,1)^{\mathrm{a}}$ & $(8,1)^{\mathrm{a}}$ & $(4,1)^{\mathrm{a}}$ \\
& Rock & $(4,1)^{\mathrm{a}}$ & $(35,5)^{\mathrm{a}}$ & $(20,3)^{\mathrm{a}}$ \\
\hline
\end{tabular}

Note: ${ }^{\mathrm{a}}(\mathrm{N} 1, \mathrm{~N} 2)$; N1: No. of Haulers and N2: No. of Loaders. 
Table 3. Comparison of cost results per stage (2012 values)

\begin{tabular}{|c|c|c|c|c|}
\hline & $\begin{array}{l}\text { Soil } \\
\text { Type }\end{array}$ & Stage 1 & Stage 2 & Stage 3 \\
\hline \multirow{3}{*}{ 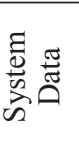 } & Moraine & $\$ 188,371^{b}$ & $\$ 3,387,394^{b}$ & $\$ 1,644,787^{b}$ \\
\hline & Granular & $\$ 118,515^{\mathrm{b}}$ & $\$ 1,604,539^{\mathrm{b}}$ & $\$ 989,070^{b}$ \\
\hline & Rock & $\$ 571,092^{b}$ & $\$ 17,267,860^{\mathrm{b}}$ & $\$ 7,408,933^{b}$ \\
\hline \multirow{3}{*}{ 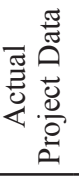 } & Moraine & $\$ 181,706^{c}$ & $\$ 2,896,625^{\mathrm{c}}$ & $\$ 1,461,258^{c}$ \\
\hline & Granular & $\$ 101,385^{c}$ & $\$ 1,601,977^{\mathrm{c}}$ & $\$ 889,892^{c}$ \\
\hline & Rock & $\$ 557,108^{c}$ & $\$ 14,438,224^{c}$ & $\$ 6,925,809^{c}$ \\
\hline
\end{tabular}

Notes: ${ }^{\text {b}}$ Values were obtained by extracting volume of soil and multiplying with productivity unit cost.

${ }^{c}$ Values were obtained from actual project data and adjusted to account for inflation.

Prior to comparison of results, it is important to note that these optimum fleet obtained are for earthwork activities only. Obtained results are compared to actual project data and found in good agreement with a percentage difference ranging between $4-16 \%$ approximately. The discrepancy between the results are based on the availability of resources at the time of construction and the assumptions made, which may not fully be compatible with the actual status of the site. For instance, tire penetration is assumed to be 3 inches. If this value is to change, the optimum fleet would be instantly affected. Also, the productivity is estimated based on an off-site methodology. Moreover, the results are based on the combined optimization of cost and time analysis of the selected fleet without taking into consideration any constraints associated with these two parameters. Overall results show that the accuracy of the system varies depending on the soil type, tire penetration, altitude, travel time, and project duration. Table 3 summarizes the costs, in present dollars, of each stage based on the type of soil.

Economical optimization of equipment fleet operating on diverse types of soils proved the workability of the system. Moreover, multiple assumptions made in this case project had significant impacts on system results. Once completed, the user is guided towards the linear scheduling optimization process. At first, productivity rates obtained from the previous process is automatically exported and plotted as a histogram. In order to effectively shape a linear schedule, a normal distribution function is fitted into the histograms and subsequently normalized to calculate expected duration. Following the estimation of earthmoving activities expected durations; the simulated annealing algorithm is deployed such that a line of balance is generated based on productivity rates extracted from the fleet productivity optimization module as illustrated in Figure 3.

Afterwards, a global optimization procedure is employed for a dataset scatter which comprises maxima and minima polynomial trends. It is important to note that representing a given dataset with a definitive polynomial is a multifaceted task since diverse parameters and co-

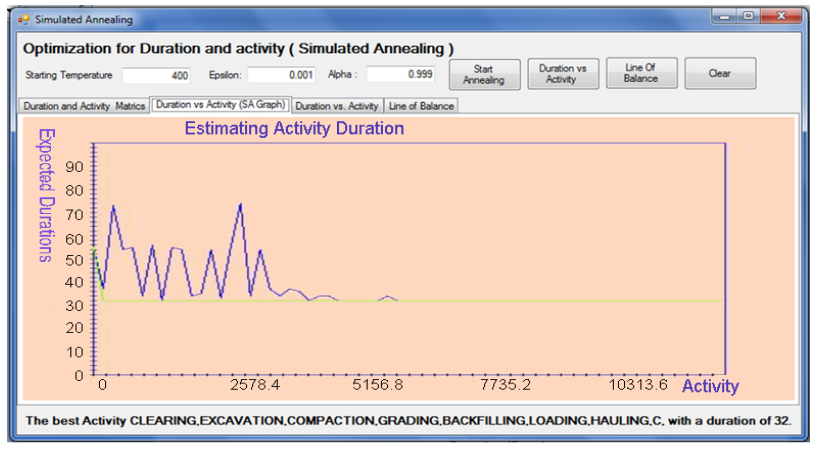

Fig. 3. Productivity rates

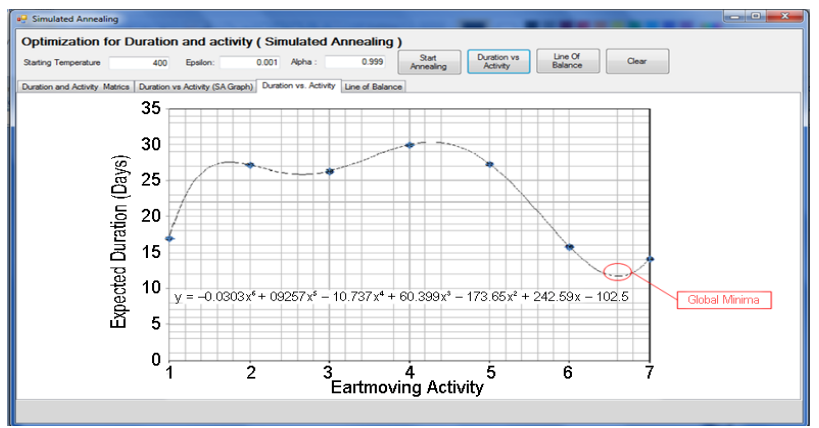

Fig. 4. Polynomial function

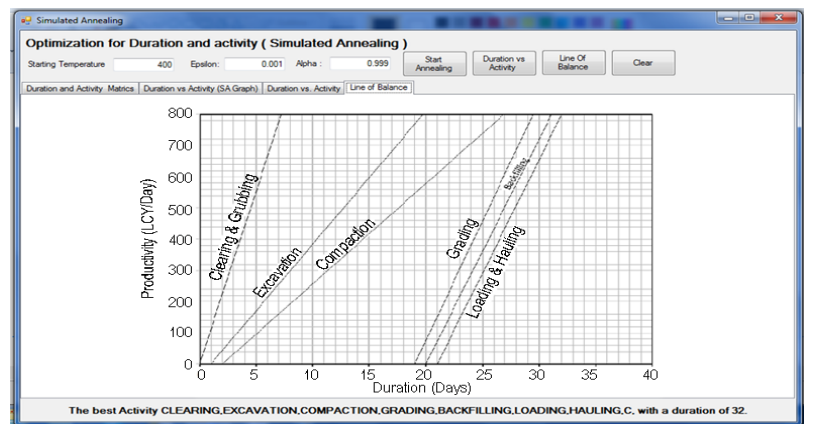

Fig. 5. Optimum line

efficients typically determine the type and degree of a function. In this study, polynomial functions of various degrees are tested based on the provenance of dataset points and their corresponding accuracy. As a result, a polynomial function of the sixth degree is found to be the most descriptive of the given dataset based on an interpolation curve that approximates a global optimum as shown in Figure 4.

Towards the end, a corresponding optimum line of balance schedule is achieved with an overall optimized duration of 32 days as illustrated in Figure 5. It is important to note that in case a scheduling conflict arises, the proposed system automatically notifies the user to modify the starting time of the preceding and/or following activity.

Typically, fleet productivity rates are not fixed and are subject to daily and possibly hourly fluctuations due 
Table 4. Comparison of expected duration (days) results per earthmoving activity

\begin{tabular}{lccccccc}
\hline \multicolumn{1}{c}{ Activity } & $\begin{array}{c}\text { Clearing \& } \\
\text { Grubbing }\end{array}$ & Excavation & Compaction & Grading & Backfilling & $\begin{array}{c}\text { Loading \& } \\
\text { Hauling }\end{array}$ & $\begin{array}{c}\text { Total } \\
\text { Duration }\end{array}$ \\
\hline System Data & 7 & 20 & 26 & 29 & 31 & 32 & $\mathbf{3 2}$ \\
\hline $\begin{array}{l}\text { Actual } \\
\text { Project Data }\end{array}$ & 8 & 24 & 31 & 34 & 36 & 39 & $\mathbf{3 9}$ \\
\hline
\end{tabular}

to unanticipated occurrences. Hence, it is uncommon to represent a line of balance as a straight line; however, in many cases owners, designers, and construction managers prefer such representation for indicative purposes at conceptual design stages. This means that productivity over a short duration might be considerably below that which is anticipated and at other times will far exceed the expected production rate. It is important to note the time buffer that separates the activities as illustrated in Figure 5. The presence of a time buffer is an acknowledgement that the timing of activities occurrences cannot be scheduled with identifying accuracy. Moreover, as shown in Figure 5, when fleet productivity of either compaction or loading activity exceeds 900 LCY/day (LCY; loose cubic yards), a diminishing effect is prominent and a scheduling conflict arises where additional time buffer is required to resolve the issue. Obtained results are compared to actual project data and found in good agreement with a percentage difference ranging between $12-18 \%$ approximately. The discrepancy between the results is due to the multiple assumptions made as well as to the availability of resources at the time of construction besides consistent productivity rates without fluctuations. For instance, fleet productivity is based on normal job site conditions. If unforeseen circumstances occur, the optimum line of balance would be instantly affected. Also, the productivity is estimated based on an off-site methodology. Moreover, the results are based on the combined optimization of cost and time analysis of the selected fleet without taking into consideration any constraints associated with these two parameters. Overall results show that the accuracy of the system varies depending on the fleet productivity obtained and availability of resources besides logistic considerations. Table 4 summarizes individual activity durations as extracted from the system optimization module.

\section{Summary, conclusions, and future work}

In summary, the proposed expert system is developed to assist owners, designers, and construction managers plan for heavy earthmoving operations. The operation analysis of different types of equipment is carried out to support economical equipment selection and develop optimized linear schedules for diverse earthmoving activities. The system is then validated through a case project selected from the literature and its outputs are compared with the actual case project data. It is apparent that the types of soil and user entries highly influence the accuracy of the developed system. Also, it is concluded that the system possess some limitations with regards to providing optimum fleet results involving rock material. That, in fact, requires much more complex studies to be conducted in order to optimize the required fleet for this type of work; such as earthwork involving mining construction. It is necessary to mention that the optimization system is developed as an estimation tool that can be used to estimate the required fleet for a particular construction project. Estimation results are based on user entries and are homogenous in type. The homogeneity of optimum results is one major limitation of the developed system. Moreover, output results of the hauling-loading complex activity are usually influenced by the availability of a spare hauler in case of a break-down event. In this study, a spare hauler is considered to be available regardless of unforeseen circumstances and is; therefore, a second limitation of the proposed system. Presently, the proposed system provides the optimum fleet that is capable of performing the excavation job of materials except for rock material. In addition, results presented in this study are anticipated to be of major significance to the construction industry and would contribute to the database of fleet management systems. This is an ongoing research and its authors are working on strengthening the system by enhancing the decision support tool and by incorporating additional optimization parameters and constraints to it. This will be done by considering more equipment specifications data and equipment alternatives. For instance, this system is limited to one major heavy equipment manufacturer, where other types of equipment manufacturers will be included. Furthermore, more specific factors that can be applied for specific types of equipment (i.e. scrapers) will be considered.

In general, it is not possible for the proposed system to predict fleet selection accurately and without errors. This is simply due to the fact of the nature and characteristics of construction projects. The main contribution of this study is the development of the computer system to optimize heavy equipment selection based on their economical operation analysis. This paper is the first attempt to incorporate economical equipment operation analysis as the framework of the fleet optimization system. The main privilege of the proposed system is the ability to automate user input data interface with heavy equipment operation analysis. The proposed system can be utilized even in projects that involve a large volume of earthwork. This capability gives the system great advantage over other optimization algorithms, prototypes, or models published in literature. 


\section{References}

Beißert, U.; Konig, M.; Bargstadt, H. 2007. Constraint-based simulation of outfitting processes in building engineering, in $24^{\text {th }}$ W78 Conference, 26-29 June 2007, Maribor, Slovenia. $7 \mathrm{p}$.

Caterpillar $^{\circledR}$. 2011. Caterpillar performance handbook. Peoria: Caterpillar. 1113 p.

Dreo, J.; Petrowski, A.; Siarry, P.; Taillard, E. 2006. Metaheuristics for hard optimization: methods and case studies. France: Springer. 372 p.

Gould, H.; Tobochnik, J. 2010. Statistical and thermal physics: with computer applications. Princeton University Press. $552 \mathrm{p}$.

Fu, J. 2013. Logistics of earthmoving operations- simulation and optimization: Licentiate thesis in Transport Science. KTH Royal Institute of Technology, Sweden.

Jrade, A.; Markiz, N.; Albelwi, N. 2012. An economical operation analysis optimization model for heavy equipment selection, International Scholarly and Scientific Research \& Innovation 6: 1223-1228.

Jrade, A.; Markiz, N. 2012. A decision-support model utilizing a linear cost optimization approach for heavy equipment selection, in Construction Research Congress, 21-23 May 2012, West Lafayette, Indiana, United States. ASCE, $100-109$.

Marzouk, M. 2002. Optimizing earthmoving operations using computer simulation: $\mathrm{PhD}$ thesis. Concordia University, USA.
Marzouk, M.; Moselhi, O. 2003. Object-oriented simulation model for earthmoving operations, Journal of Construction Engineering and Management 129(2): 173-181.

http://dx.doi.org/10.1061/(ASCE)0733-9364(2003)129:2(173)

Marzouk, M.; Moselhi, O. 2004. Multiobjective optimization of earthmoving operations, Journal of Construction Engineering and Management 130(1): 105-113.

http://dx.doi.org/10.1061/(ASCE)0733-9364(2004)130:1(105)

Moselhi, O.; Marzouk, M. 2000. Automated system for cost estimating of earthmoving operations, in Proc. of the $17^{\text {th }}$ International Symposium on Automation and Robotics in Construction (ISARC), 18-20 September 2000, Taipei, Taiwan, 1053-1058.

Rachmat, F.; Song, L.; Lee, S. H. 2012. Stochastic look-ahead scheduling method for linear construction projects, Journal of Risk Analysis and Crisis Response 2(4): 252-260. http://dx.doi.org/10.2991/jrarc.2012.2.4.4

Rafsanjani, H. 2011. A computer model for selecting equipment in earth-fill dam projects, Journal of Civil Engineering and Architecture 5(5): 465-470.

Rossi, F.; Van Beek, P.; Walsh, T. 2006. Handbook of constraint programming. USA: Elsevier. 978 p.

Shapira, A.; Goldenberg, M. 2005. AHP-based equipment selection model for construction projects, Journal of Construction Engineering and Management 131(12): 1263-1273. http://dx.doi.org/10.1061/(ASCE)0733-9364(2005) $131: 12(1263)$

Nizar MARKIZ. PhD candidate in the Construction, Engineering and Infrastructure Management in the Faculty of Graduate and Postdoctoral Studies at the Ottawa-Carleton Institute of Civil Engineering. His research interests include integrating decision support system with BrIM, fleet, time, and cost optimization for bridges at the conceptual design stage.

Ahmad JRADE. Assistant Professor of Construction Engineering and Management at the University of Ottawa, Department of Civil Engineering. His research interests include computer integrated construction, 3D-CAD Modeling, Management Information System (MIS) and Life Cycle Cost Analysis. He developed a management information system that integrates 3D-CAD drawings with cost databases to generate conceptual cost estimates in order to forecast future costs and income for proposed building projects based on their size and budget. 